\title{
Effects of Positive End-Expiratory Pressure, Inhaled Nitric Oxide and Surfactant on Expression of Proinflammatory Cytokines and Growth Factors in Preterm Piglet Lungs
}

\author{
LILING QIAN, HAIPEI LIU, WENLIANG YU, XIAONING WANG, ZHONGHOU SUN, WEI WANG, LIEWEI ZHU, AND BO SUN
}

Laboratory of Pediatric Respiratory and Critical Care Medicine, Fudan University, Shanghai 200032, P.R. China

\begin{abstract}
We hypothesized that imbalance of proinflammatory cytokines and growth factors (GFs) in immature lungs of early postnatal life may be affected by protective ventilation strategy, and evaluated correlations of these aspects. Preterm neonate piglets were mechanically ventilated with low tidal volume and 5-6 or $10-12 \mathrm{~cm}$ $\mathrm{H}_{2} \mathrm{O}$ positive end-expiratory pressure (PEEP) with or without surfactant and inhaled nitric oxide (iNO) for $6 \mathrm{~h}$, followed by biochemical, biophysical, and histopathological assessment of lung injury severity. Compared with surfactant and the control, iNO combined with lower PEEP exerted better oxygenation, lower activity of myeloperoxidase, lower expression of mRNA of interleukin (IL)-1 $\beta$, IL-6, IL-8, and platelet derived growth factor-B (PDGF-B), but higher expression of insulin-like growth factor-I (IGF-I), whereas that of tumor necrosis factor- $\alpha$, keratinocyte GF, hepatocyte GF, vascular endothelial growth factor, and TGF- $\beta 1$ had no or modest changes. IL- $1 \beta$, IL- 6 mRNA were closely correlated to PDGF-B mRNA and myeloperoxidase, but inversely to IGF-I mRNA, $\mathrm{PaO}_{2} /$ $\mathrm{FiO}_{2}$ and dynamic lung compliance at $6 \mathrm{~h}$. These results indicate that the association of lower PEEP and iNO may be more protective than surfactant on preventing lung injury and facilitating reparation by affecting the expression of proinflammatory cytokines and GFs.

(Pediatr Res 64: 17-23, 2008)
\end{abstract}

$I^{\text {nem }}$ mmature lungs of neonates in early postnatal life are vulnerable to mechanical ventilation and oxygen therapy (1). The adverse effects of these therapies involve inflammatory damage because of alveolar overstretching or hyperoxia, and are related to an imbalance between injury severity and reparation potential of the lung tissue (2). Furthermore, inflammatory mediators may interfere with alveolarization in neonatal lung development (3), and its pathologic consequence is bronchopulmonary dysplasia, or chronic lung disease of prematurity (4). Alteration of de novo synthesis of several growth factors (GFs) in the lungs is vital in injury-repair response and remodeling. Among them, platelet derived growth factor-B (PDGF-B), insulin-like growth factor-I (IGF-I), vascular endothelial growth factor (VEGF), TGF- $\beta 1$, keratinocyte

Received August 27, 2007; accepted February 20, 2008.

Correspondence: Bo Sun, M.D., Ph.D., Children's Hospital of Fudan University, 183 Feng Lin Road, Shanghai 200032, P.R. China; e-mail: bsun@shmu.edu.cn or qyang75@yahoo.com.cn

This work was supported by grants from the National Natural Science Foundation (No. 30170989, 30571974) and Shanghai Municipal Commissions of Education (02SG02) and Science and Technology (B.S.); Young Investigator Award of Fudan University Shanghai Medical College (No. 05-Y-09, L.Q.). China Medical Board (03-786)

Drs. Liling Qian and Haipei Liu equally contributed to the main part of this study as their theses work.

Supplemental material available online at www.pedresearch.org
(KGF), and hepatocyte growth factor (HGF) mediate tissue interactions and regulate a variety of cellular functions that are crucial for fetal lung development, especially in septation and alveolarization (5). However, little is known about their postnatal response to clinical intervention with mechanical ventilation and oxygen therapy. ${ }^{1}$

Applying adequate ventilation strategy should minimize ventilator-induced lung injury. Naik et al. (6) found the use of positive end-expiratory pressure (PEEP) at $4 \mathrm{~cm} \mathrm{H}_{2} \mathrm{O}$ minimized alveolar protein leakage and neutrophil infiltration in surfactant-treated preterm lambs compared with that of PEEP at $7 \mathrm{~cm} \mathrm{H}_{2} \mathrm{O}$. However, there are few studies reporting effects of PEEP on expression of proinflammatory cytokines and GFs in the immature lungs and the mechanisms underlying their interactions. Furthermore, surfactant and inhaled nitric oxide (iNO) are two alternative therapies for neonates suffering from severe hypoxemic respiratory failure. Recently, experimental studies showed that NO may play roles in enhancement of pulmonary surfactant activity (7), inhibition of neutrophil infiltration and retention (8), inhibition of cytokine release (9), modulation of lung growth (10), and especially mitigation of lung injury associated with fibrin deposition in alveolar space (11). Based on previous experimental studies, surfactant and iNO exerted better effects than either treatment alone in protection of mature pig lungs from acute injury (12-13). Our aim in the present study was to use a ventilated premature piglet model (14) and test a hypothesis that two levels of PEEP, in the presence of iNO and/or surfactant, should affect expression of proinflammatory cytokines and GFs differently, and delineate correlations of these factors in response to these therapies.

\section{METHODS}

Management and mechanical ventilation of animals. Animal study protocols were approved by the Scientific and Ethics Committees at Children's Hospital of Fudan University. Preterm piglets at gestational age (GA) of 97-100 d (term 114 d) were obtained by cesarean section (14). A tracheotomy was performed with placement of an endotracheal tube. The piglets were then ventilated individually and simultaneously with Servo 300 or 900C (SiemensElema, Solna, Sweden). The ventilators were initially set at a frequency of 50 breaths/min, peak inspiratory pressure of $20-30 \mathrm{~cm} \mathrm{H}_{2} \mathrm{O}$, PEEP of 5-6 cm $\mathrm{H}_{2} \mathrm{O}$, inspiration time of $0.4 \mathrm{~s}$, and fraction of inspired oxygen $\left(\mathrm{FiO}_{2}\right)$ of $0.8-1.0$. The ventilator settings were adjusted to provide a tidal volume of

Abbreviations: GF, growth factor; iNO, inhaled NO; PEEP, positive endexpiratory pressure 
$6-8 \mathrm{~mL} / \mathrm{kg}$ and to target $\mathrm{PaO}_{2}$ at $50-80 \mathrm{~mm} \mathrm{Hg}$, and $\mathrm{PaCO}_{2}$ at $30-50 \mathrm{~mm} \mathrm{Hg}$ whereas PEEP was kept constant.

Experimental design. After initial stabilization for $15 \mathrm{~min}$ of ventilation, those animals with $\mathrm{PaO}_{2} / \mathrm{FiO}_{2} \leq 250 \mathrm{~mm} \mathrm{Hg}$ were enrolled and randomly assigned to groups as either low PEEP at 5-6 $\mathrm{cm} \mathrm{H}_{2} \mathrm{O}$ (PEEP 5) or high PEEP at $10-12 \mathrm{~cm} \mathrm{H}_{2} \mathrm{O}$ (PEEP 10), and immediately allocated to different subgroups $(n=6)$ receiving surfactant and/or iNO. The subgroups are defined in PEEP 5: C5, control; PS5, surfactant; NO5, iNO; SNO5, both surfactant and iNO; whereas those in PEEP 10: C10, control; PS10, surfactant; NO10, iNO; SNO10, both surfactant and iNO. A porcine lung surfactant (Curosurf) was used for the PS and SNO subgroups at $100 \mathrm{mg} / \mathrm{kg}$ body weight instilled in bolus into the lungs via the endotracheal tube. NO gas was continuously supplied to ventilator circuit at 10 parts per million in volume (ppm) during the whole ventilation (12).

Blood gas analysis and measurement of lung mechanics. Before ventilation was initiated, blood samples were taken for baseline of arterial blood gas values (ABL-5, Radiometer, Copenhagen, Denmark). After stabilizing for $15 \mathrm{~min}$, lung mechanics and blood gas values were recorded and this time was designated as treatment time $0 \mathrm{~h}$. Dynamic compliance of respiratory system (Cdyn) and airway resistance (Raw) were measured with a pneumotachometer (Navigator, Newport Beach, CA). These parameters were measured at each hour up to $6 \mathrm{~h}$ or until animal death. Values of $\mathrm{PaO}_{2} / \mathrm{FiO}_{2}$ and oxygenation index [OI, = mean airway pressure $\left(\right.$ in $\left.\mathrm{cm} \mathrm{H}_{2} \mathrm{O}\right) \times \mathrm{FiO}_{2} \times 100 / \mathrm{PaO}_{2}$ (in $\mathrm{mmHg}$ )] were calculated. Blood methemoglobin (MetHb) and nitrite/nitrate $\left(\mathrm{NO}_{2}{ }^{-} / \mathrm{NO}_{3}{ }^{-}\right)$were measured according to routine methods (12).

Processing of lungs. At the end of the experiment, the piglets were killed with $10 \%$ potassium chloride i.v. After inspection of pneumothorax, the thorax was opened, and the lungs and heart were removed en bloc and lung tissue from the tip of right mid-lobe was used for determining wet-to-dry weight ratio (W/D) (12). The rest of right mid-lobe was immediately frozen in liquid nitrogen for further measurement of malondialdehyde (MDA) content, myeloperoxidase (MPO) activity, NOS activity, and RNA isolation. Bronchoalveolar lavage of the right lung was performed with sterile saline at $4^{\circ} \mathrm{C}$ via intratracheal tube, and total cell counts of bronchoalveolar lavage fluid (BALF) were determined with a standard hemocytometer. The left lung was perfusion fixed for 30 min via pulmonary artery with $4 \%$ paraformaldehyde (13-14).

Biochemical and biophysical analysis of BALF contents. Total phospholipids (TPL) were isolated from chloroform-methanol (2:1) extracts of BALF, then disaturated phosphatidylcholine (DSPC) was recovered by neutral alumina column chromatography after exposure of lipid extract to osmium tetroxide, and phospholipids were quantified by phosphorus assay according to a routine procedure $(12,14)$. Total proteins (TP) were measured by Lowry's method as described before (14). The DSPC and TP ratio from BALF was for evaluation of alveolar surfactant pool $v s$. vascular-to-alveolar protein leakage. Minimum and maximum surface tension $\left(\gamma_{\min }\right.$ and $\left.\gamma_{\max }\right)$ of TPL from BALF were measured with a pulsating bubble surfactometer (Electronetics, Buffalo, NY) (13-14).

Measurement of MDA content, MPO and NOS activity in lung tissues. MDA content and MPO activity were assessed for lipid peroxidation and leukocyte infiltration in lung tissue, respectively, as previously described (12). Protein content in samples was measure by Lowry's method as described above. NOS activity was determined through the conversion of L- $\left[{ }^{3} \mathrm{H}\right] \operatorname{arginine}$ to $\mathrm{L}-\left[{ }^{3} \mathrm{H}\right]$ citrulline based on modifications of previously described methods (15).

Lung histopathology and morphometry. Sections of $5-\mu \mathrm{m}$ thickness, stained with hematoxylin and eosin, were examined under light microscopy for lung histopathologic changes (13). Lung expansion was quantified with a point-counting method, and expressed as volume density ( $\mathrm{Vv})$ of aerated alveolar spaces, using total parenchyma as a reference volume. Fifty fields of each animal lung were examined at $\times 300$ magnification and field-to-field variability was determined by calculating the coefficient of variation of $\mathrm{Vv}$ (CV [Vv]) (13-14).

Immunohistochemistry of GFs in lung tissues. After deparaffinization and rehydration, lung sections mounted on $\alpha$-aminopropyltriethoxysilane-coated slides were incubated with rabbit polyclonal antibodies against IGF-I (1:200, PeproTech, Rocky Hill, NJ) and PDGF-B (1:100, Santa Cruz Biotechnologies, Santa Cruz, CA) or goat polyclonal antibody against KGF (1:100, Santa Cruz). Antigens were visualized using the DAKO EnVision Staining System (DAKO, Carpinteria, CA) for IGF-I and PDGF-B, or the ABC Staining System (Santa Cruz) for KGF according to the manufacturer's instructions. Negative controls were stained by substitution of the primary antibodies with nonimmune immunoglobulin. Positive staining was recognized under a microscope as brown color for diaminobenzidine.

Measurement of cytokine and GF mRNA in lung tissues by quantitative real-time PCR. Total RNA was extracted from lung tissue using TRIzole reagent and was reversely transcribed into cDNA. Gene sequence information
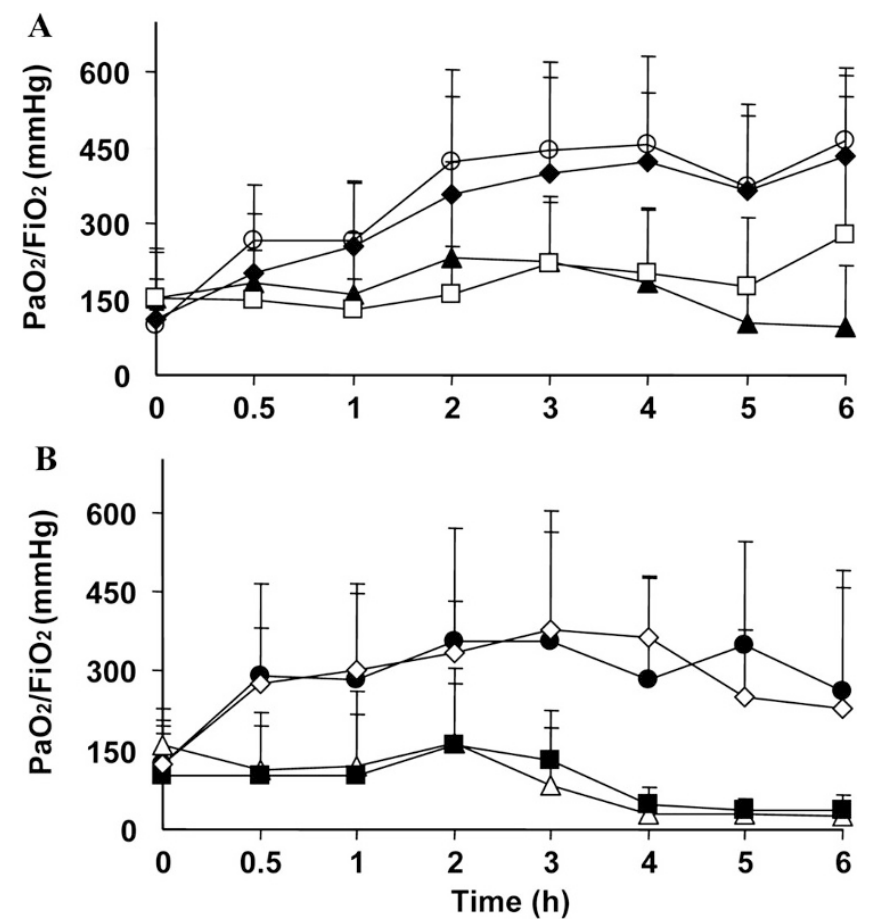

Figure 1. Changes of $\mathrm{PaO}_{2} / \mathrm{FiO}_{2}$ during ventilation time (A, PEEP 5 [5-6 cm $\mathrm{H}_{2} \mathrm{O}$ ] and $B$, PEEP 10 [10-12 $\mathrm{cm} \mathrm{H}_{2} \mathrm{O}$ ] subgroups, $n=6$ ). Subgroup definitions: $(\mathbf{\Delta})$ C5, control with PEEP 5; $\square$ ) PS5, PEEP $5+$ surfactant; $(\bigcirc)$ NO5, PEEP $5+$ iNO; $(\checkmark)$ SNO5, PEEP $5+$ surfactant + iNO; $(\triangle)$ C10, control with PEEP 10; (ם) PS10, PEEP $10+$ surfactant; $(\bullet)$ NO10, PEEP $10+\mathrm{iNO} ;(\diamond)$ SNO10, PEEP $10+$ surfactant + iNO. In $A$, at $4 \mathrm{~h}$, NO5 vs. C5 and PS5, $p<0.05$; at 5 and $6 \mathrm{~h}$, NO5 and SNO5 vs. C5, $p<$ 0.05. In $B$, at 4 and $5 \mathrm{~h}, \mathrm{NO} 10$ and SNO10 vs. C10 and PS10, $p<0.05$ or $<0.01$; at 4 and 5 h, C5 vs. C10, and PS5 vs. PS10, $p<0.05$.

Table 1. General conditions and blood gas values before ventilation

\begin{tabular}{lccccccc}
\hline Subgroup & $\mathrm{Sex}(\mathrm{M} / \mathrm{F})$ & $\mathrm{GA}(\mathrm{d})$ & $\mathrm{BW}(\mathrm{g})$ & $\mathrm{PaO}_{2}(\mathrm{~mm} \mathrm{Hg})$ & $\mathrm{PaCO}_{2}(\mathrm{~mm} \mathrm{Hg})$ & $\mathrm{pH}$ & $\mathrm{BE}(\mathrm{mmol} / \mathrm{L})$ \\
\hline C5 & $3 / 3$ & $98.3 \pm 1.2$ & $742 \pm 139$ & $33.2 \pm 9.1$ & $56.7 \pm 18.9$ & $7.13 \pm 0.20$ & $-10.7 \pm 10.3$ \\
NO5 & $3 / 3$ & $98.7 \pm 1.2$ & $757 \pm 151$ & $21.7 \pm 3.6$ & $90.2 \pm 24.8$ & $7.08 \pm 0.15$ & $-10.3 \pm 8.3$ \\
PS5 & $4 / 2$ & $99.0 \pm 0.9$ & $823 \pm 150$ & $22.8 \pm 2.5$ & $92.7 \pm 21.9$ & $7.00 \pm 0.13$ & $-11.8 \pm 3.1$ \\
SNO5 & $3 / 3$ & $98.5 \pm 0.8$ & $692 \pm 240$ & $25.5 \pm 6.7$ & $88.0 \pm 33.9$ & $7.06 \pm 0.24$ & $-10.0 \pm 11.7$ \\
C10 & $3 / 3$ & $98.0 \pm 1.1$ & $705 \pm 91$ & $24.2 \pm 8.1$ & $63.0 \pm 19.0$ & $7.18 \pm 0.10$ & $-5.7 \pm 10.3$ \\
NO10 & $2 / 4$ & $98.2 \pm 1.0$ & $777 \pm 125$ & $25.3 \pm 3.5$ & $77.5 \pm 11.9$ & $7.14 \pm 0.10$ & $-6.0 \pm 6.0$ \\
PS10 & $4 / 2$ & $98.3 \pm 1.0$ & $752 \pm 129$ & $20.0 \pm 10.9$ & $83.8 \pm 16.3$ & $7.07 \pm 0.10$ & $-8.8 \pm 5.7$ \\
SNO10 & $4 / 2$ & $98.2 \pm 1.0$ & $810 \pm 209$ & $19.3 \pm 4.8$ & $99.0 \pm 9.3$ & $7.00 \pm 0.02$ & $-13.0 \pm 1.3$ \\
\hline
\end{tabular}

PEEP 5, positive end-expiratory pressure of 5-6 $\mathrm{cm} \mathrm{H}_{2} \mathrm{O}$; PEEP 10, 10-12 $\mathrm{cm} \mathrm{H}_{2} \mathrm{O}(n=6$ in each subgroup); C5, control in PEEP 5; PS5, PEEP $5+$ surfactant; NO5, PEEP 5 + iNO; SNO5, PEEP 5 + surfactant + iNO; C10, control in PEEP 10; PS10, PEEP 10 + surfactant; NO10, PEEP 10 + iNO; SNO10, PEEP $10+$ surfactant + iNO. GA, gestational age; BW, birth weight; M, male; F, female; BE, base excess. 
of interleukin (IL)- $1 \beta, 6,8$, tumor necrosis factor- $\alpha$ (TNF- $\alpha$ ), PDGF-B, IGF-I, KGF, HGF, VEGF, and TGF- $\beta 1$ was obtained using nucleotide databases (www.ncbi.nlm.nih.gov/Genbank/index.html), and primer sequences and optimal PCR conditions are listed in Table A (supplemental material online at www.pedresearch.org). Traditional PCR was performed to obtain standards for quantitatively assessing mRNA copy numbers. Real-time PCR was performed using an ABI PRISM 7000 system (Applied Biosystems, Foster City, CA). The amplification was carried out in a $25 \mu \mathrm{L}$ reaction volume containing $12.5 \mu \mathrm{L}$ SYBR Green I master mix (TOYOBO, Osaka, Japan). Copy numbers of the gene in samples were determined from the standard curve. Glyceraldehyde phosphate dehydrogenase was used as a housekeeping gene for the analysis of the genes of interest. The relative quantities of mRNA are presented as common logarithm of the copy numbers of targeted gene/copy numbers of glyceraldehyde phosphate dehydrogenase $\left(\times 10^{5}\right)$.

Statistical analysis. All the continuous data are presented as means and SD. Data analysis of subgroups was performed separately in each of the clustered PEEP groups. Variables were subjected to ANOVA or KruskalWallis test for differences among the subgroups, followed by post hoc test of Bonferroni or Wilcoxon-Mann-Whitney test for between-group differences, and by repeated measures or Wilcoxon signed rank test for within-group differences (changes with time). The comparison for subgroups with the same treatment between the PEEP 5 and PEEP 10 was carried on by Student-Newman-
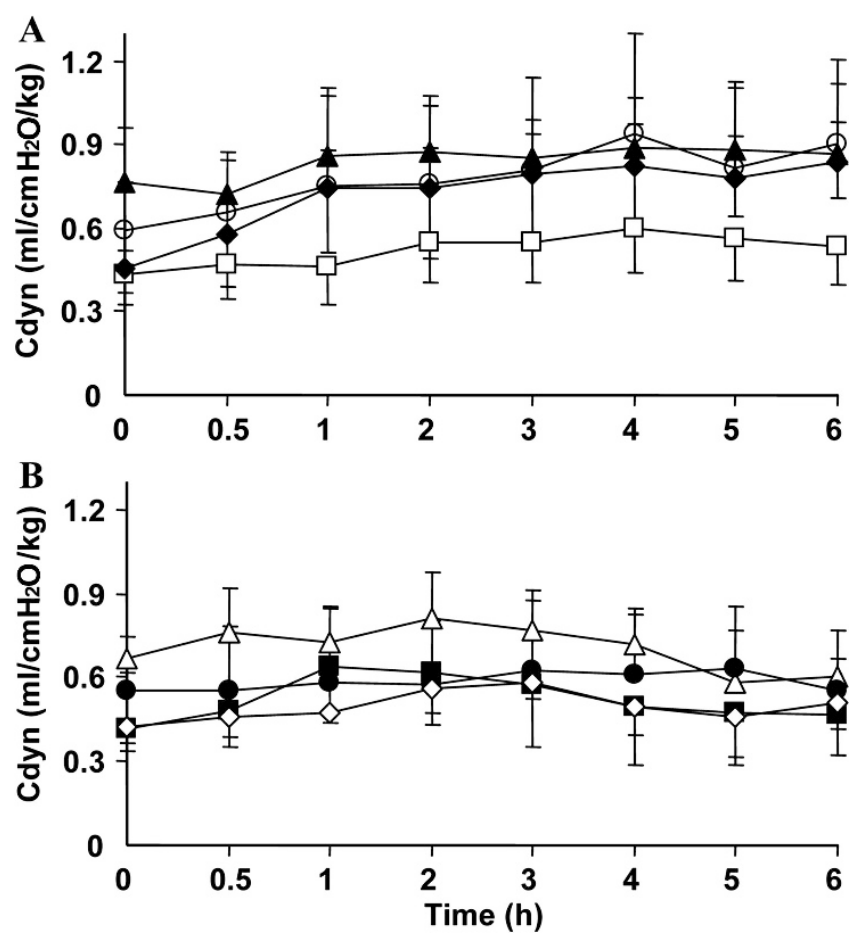

Figure 2. Changes of dynamic compliance (Cdyn) during ventilation time ( $A$, PEEP 5 subgroups; $B$, PEEP 10 subgroups, $n=6$ ). Subgroup definitions see Figure 1 legends. PS5 and SNO5 vs. C5, PS10 and SNO10 vs. C10 at 0 h, $p<$ 0.01 ; C5 vs. C10 at $5 \mathrm{~h}$, and SNO5 vs. SNO10 at 1, 4, 5 and $6 \mathrm{~h}, p<0.05$.
Keuls test or Mann-Whitney test. Pearson correlation test was used for correlations between two parameters. A $p$ value $<0.05$ was regarded as statistically significant.

\section{RESULTS}

General conditions of the animals. There were no intergroup differences in age, body weight, sex, and baseline condition of blood gas values before the mechanical ventilation was initiated (Table 1), but severe hypoxemia, hypercapnia, and acidosis were present. All the animals in the PEEP 5 subgroups survived $6 \mathrm{~h}$ of the ventilation period, whereas in C10 three died after $5 \mathrm{~h}$, and in NO10, PS10, and SNO10, one each died after $5 \mathrm{~h}$.

Oxygenation. There was a trend of lower $\mathrm{FiO}_{2}$ requirement in the NO-treated subgroups (Table B, supplemental material online at www.pedresearch.org). When combining the values from NO10 and SNO10, it revealed significant difference compared with that from C10 and PS10 at 2-6h $(p<0.05)$. Sustained and significant improvement in $\mathrm{PaO}_{2} / \mathrm{FiO}_{2}$ was found in NO5 and SNO5, as corroborated by repeated measures as well, whereas only modest improvement in PS5 and no improvement in $\mathrm{C} 5$ after $6 \mathrm{~h}$ treatment were found (Fig. 1A). In the subgroups of PEEP 10, there was sustained improvement in $\mathrm{PaO}_{2} / \mathrm{FiO}_{2}$ in $\mathrm{NO10}$ and $\mathrm{SNO} 10$ in contrast to deterioration in C10 and PS10 (Fig. 1B). Furthermore, the improvement was more obvious in the PEEP 5 subgroups than in the PEEP 10 (Fig. 1). The differences were seen in $\mathrm{PaO}_{2} / \mathrm{FiO}_{2}$ between $\mathrm{C} 5$ and $\mathrm{C} 10$, as well as in PS5 and PS10 at 4 and $5 \mathrm{~h}(p<0.05)$. The changes of OI with time were similar to that of $\mathrm{PaO}_{2} / \mathrm{FiO}_{2}$ in both PEEP 5 and PEEP 10 subgroups.

Lung mechanics. In both PEEP 5 and PEEP 10 subgroups, lower Cdyn was seen in PS5, SNO5, PS10 and SNO10 compared with C5 and C10 at $0 \mathrm{~h}$ of treatment $(p<0.01)$, but not between the PEEP 5 and PEEP 10 subgroups with the same treatment. During the treatment, it was significantly improved in both NO5 and SNO5 compared with C5, but not in any of the PEEP 10 subgroups (Fig. $2 A$ and $B$ ). Significant differences were found in Cdyn between $\mathrm{C} 5$ and $\mathrm{C} 10$ at $5 \mathrm{~h}(p<$ $0.05)$, and between SNO5 and SNO10 at 1, 4, 5, and $6 \mathrm{~h}(p<$ 0.05 or $<0.01$ ). Raw in PEEP 10 subgroups were lower than the corresponding ones of the PEEP 5, especially between NO10 and $\mathrm{NO} 5$ at $1,3,4$, and $5 \mathrm{~h}\left(37-40\right.$ vs. $80-87 \mathrm{~cm} \mathrm{H}_{2} \mathrm{O} / \mathrm{L} / \mathrm{S}, p<$ $0.05)$, or PS10 and PS5 at $3 \mathrm{~h}\left(36 v s .99 \mathrm{~cm} \mathrm{H}_{2} \mathrm{O} / \mathrm{L} / \mathrm{S}, p<0.01\right)$. The levels of MetHb (1.79-1.92\%) and $\left(\mathrm{NO}_{2}{ }^{-} / \mathrm{NO}_{3}{ }^{-}\right)$(mean

Table 2. Cytological, biochemical, and biophysical analyses of contents in BALF

\begin{tabular}{|c|c|c|c|c|c|c|c|c|}
\hline Subgroup & $\begin{array}{l}\text { Total cell counts } \\
\left(\times 10^{6} / \mathrm{mL}\right)\end{array}$ & $\mathrm{TP}(\mathrm{mg} / \mathrm{kg})$ & TPL $(\mathrm{mg} / \mathrm{kg})$ & DSPC (mg/kg) & DSPC/TPL (\%) & DSPC/TP $(\mathrm{mg} / \mathrm{mg})$ & $\gamma_{\min } \mathrm{mN} / \mathrm{m}$ & $\gamma_{\max } \mathrm{mN} / \mathrm{m}$ \\
\hline C5 & $0.93 \pm 0.49$ & $49.4 \pm 17.1$ & $22.8 \pm 6.0$ & $12.8 \pm 4.8$ & $54.7 \pm 8.8$ & $0.28 \pm 0.11$ & $13.8 \pm 1.9$ & $42.7 \pm 5.1$ \\
\hline NO5 & $1.10 \pm 0.57$ & $36.0 \pm 18.8$ & $22.3 \pm 10.0$ & $14.3 \pm 7.2$ & $63.2 \pm 10.2$ & $0.49 \pm 0.28$ & $13.0 \pm 3.5$ & $43.3 \pm 5.6$ \\
\hline PS5 & $1.58 \pm 0.77$ & $46.4 \pm 8.9$ & $63.1 \pm 20.3 \dagger \S$ & $37.4 \pm 10.0 \dagger \S$ & $60.6 \pm 8.8$ & $0.96 \pm 0.45 \dagger \dagger$ & $12.3 \pm 2.5$ & $42.0 \pm 4.1$ \\
\hline SNO5 & $1.27 \pm 0.76$ & $43.7 \pm 12.4$ & $60.6 \pm 22.5 \dagger \S$ & $36.6 \pm 11.5 \dagger \S$ & $61.9 \pm 8.5$ & $0.84 \pm 0.09 *$ & $12.0 \pm 2.4$ & $37.8 \pm 7.0$ \\
\hline $\mathrm{C} 10$ & $1.32 \pm 0.70$ & $53.7 \pm 10.2$ & $20.4 \pm 5.6$ & $11.7 \pm 2.9$ & $57.9 \pm 8.8$ & $0.22 \pm 0.06$ & $15.3 \pm 4.1$ & $45.7 \pm 3.6$ \\
\hline NO10 & $1.18 \pm 0.67$ & $44.4 \pm 8.5$ & $20.1 \pm 4.7$ & $12.2 \pm 3.7$ & $59.7 \pm 8.9$ & $0.29 \pm 0.12$ & $13.7 \pm 3.2$ & $39.2 \pm 6.9$ \\
\hline PS10 & $1.48 \pm 0.81$ & $49.1 \pm 15.8$ & $61.9 \pm 19.3 \dagger \S$ & $35.4 \pm 9.4 \dagger \S$ & $58.6 \pm 9.7$ & $0.79 \pm 0.37 \dagger \S$ & $12.7 \pm 3.3$ & $40.3 \pm 8.4$ \\
\hline SNO10 & $1.12 \pm 0.55$ & $45.5 \pm 16.6$ & $53.2 \pm 16.2 \dagger \S$ & $33.7 \pm 10.5 \dagger \S$ & $63.1 \pm 6.4$ & $0.78 \pm 0.25 \dagger \S$ & $13.3 \pm 2.8$ & $43.0 \pm 6.6$ \\
\hline
\end{tabular}

Subgroup definitions and abbreviations see table 1 legends ( $n=6$ in each subgroup). BALF, bronchoalveolar lavage fluids; TP, total proteins; TPL, total phospholipids; DSPC, disaturated phosphatidylcholine; $\gamma_{\min }, \gamma_{\max }$, minimal and maximal surface tension. In PEEP 5 subgroups $* p<0.05, \dagger p<0.01 v s$. C5 and $\ddagger p<0.05 v s$. NO5; in PEEP 10 subgroups, $\dagger p<0.01 v s$. C10, and $\S p<0.01 v s$. NO10. 
values at $115-124 \mu \mathrm{M}$ ) were not significantly different between the NO-treated and control subgroups.

Total cell counts, TP and phospholipids assay in BALF. No significant differences were found in total cell counts and TP of BALF across the subgroups (Table 2). The values of TPL and DSPC on C5 and C10 were about 20 and 12 $\mathrm{mg} / \mathrm{kg}$, respectively, suggestive of immature lungs with lower alveolar surfactant pool. Significant increases in TPL, DSPC, and DSPC/TP were found in surfactant-treated subgroups compared with controls or NO-only subgroups $(p<0.05)$, but no difference in DSPC/TPL, $\gamma_{\min }$ and $\gamma_{\max }$ of TPL among all subgroups, nor between the PEEP 5 and PEEP 10 subgroups with the same treatment.

W/D, MDA content, MPO, and NOS activity in lung tissues. W/D in NO10 was significantly lower than that in NO5 $(p<0.05)$, but no significant differences in the other subgroups (Table 3). MDA content and NOS activity showed no differences in either PEEP 5 or PEEP 10 subgroups, or between the PEEP 5 and PEEP 10 subgroups with the same treatment. MPO activities significantly decreased in NO5 and SNO5 compared with C5 $(p<0.01)$, and significant difference was seen between NO5 and NO10 $(p<0.05)$.

Lung histopathology and morphometry. Histopathologically, there was relatively less uniform aeration of alveoli with edema and neutrophil infiltration in both C5 and C10 (Fig. 3) and by $\mathrm{Vv}$ (Table 3). Lung inflammation was markedly alleviated in NO5, NO10, and SNO5 but less prominent in PS5, PS10, and SNO10 (Fig. 3). Compared with PEEP 5, lungs from the PEEP 10 were more aerated alternative to patchy overexpansion but no significant differences were found in $\mathrm{Vv}$ (Table 3).

Localization of GFs by immunostaining. By immunohistochemistry, PDGF-B and IGF-I were expressed in mesenchyme and epithelia. The PEEP 5 showed mild immunostainning of PDGF-B compared with the corresponding ones in the PEEP 10 , whereas it was more prominent in C5, PS5, C10, and PS10 than that in NO5, SNO5, NO10, and SNO10 (Fig. 4). In contrast, there was an obvious trend of decreased IGF-I expression in C10 and PS10 compared with C5 and PS5, and the immunostaining in NO5, SNO5, NO10, and SNO10 was more intense than that seen in C5, PS5, C10, and PS10 (Fig. 5). Immunostaining of KGF was located in mesenchyme and epithelial cells in airways and alveoli.

Expression of proinflammatory cytokine and GF mRNA in lung tissues. There was a trend of decreased IL-1 $\beta$ and
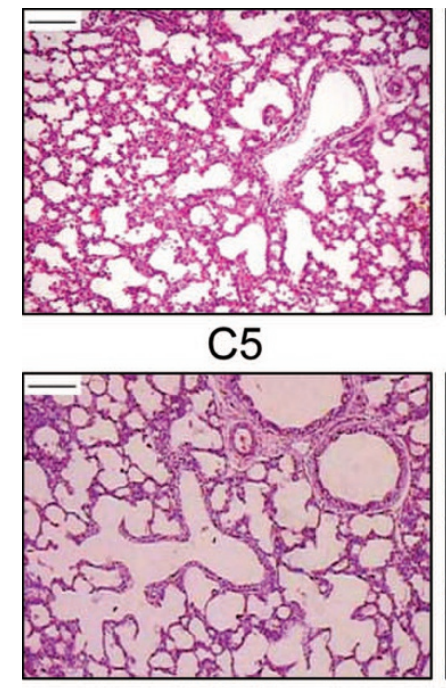

NO5

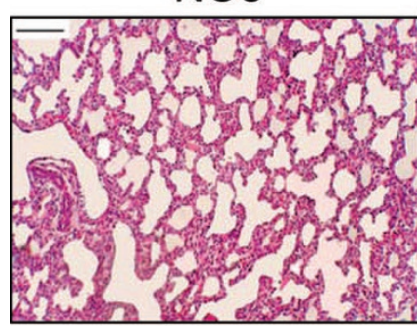

PS5

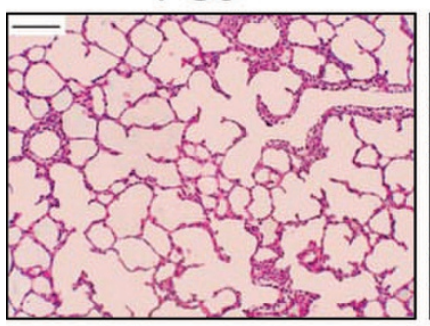

SNO5
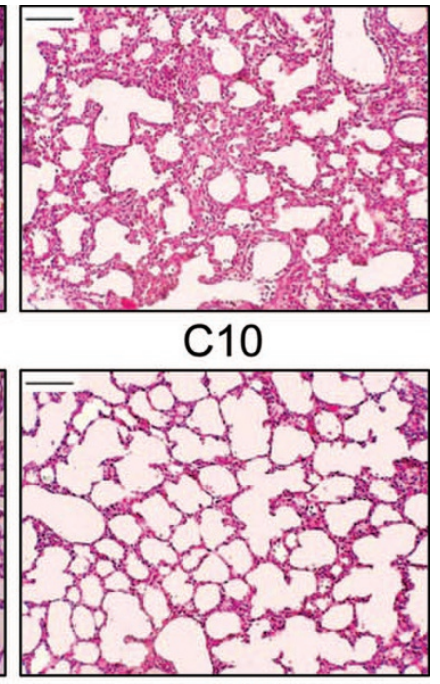

NO10

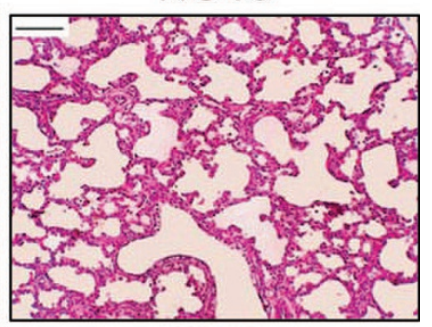

PS10

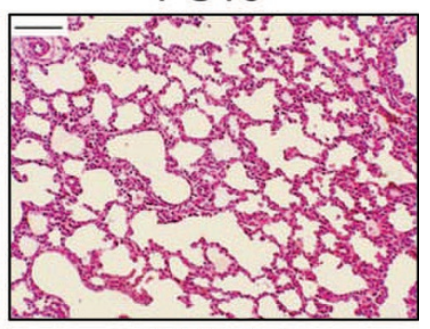

SNO10
Figure 3. Lung histopathology by light microscopy. Subgroup definitions see Figure 1 legends. Scale bar $=50 \mu \mathrm{m}$. Hematoxylin and eosin, $200 \times$.

IL-6 mRNA expression in NO5, NO10, SNO5, and SNO10 compared with C5, C10, PS5, and PS10 (Fig. 6A and $B$ ), and alleviated expression of IL- $1 \beta$ and IL-6 mRNA in the PEEP 5 subgroups than in the corresponding ones in PEEP 10. The expressions of IL-1 $\beta$ and IL-6 mRNA in both NO5 and SNO5 were lower than C5 or PS5 $(p<0.05)$, respectively. A significant decrease of IL-1 $\beta$ mRNA was seen in NO10 vs. C10 $(p<0.01)$, SNO10 vs. PS10 $(p<0.01)$ (Fig. $6 A$ and $B$ ).

Table 3. Physiological and biochemical analysis of lung tissue contents and morphometric assessment of alveolar aeration

\begin{tabular}{lccccccc}
\hline Subgroup & W/D & MDA (nmol/mg protein) & MPO (U/g tissue) & iNOS (U/mg protein) & cNOS (U/mg prot) & Vv & CV (Vv) \\
\hline C5 & $6.82 \pm 0.73$ & $1.57 \pm 0.32$ & $3.31 \pm 0.42$ & $0.18 \pm 0.08$ & $0.99 \pm 0.24$ & $0.39 \pm 0.11$ & $0.31 \pm 0.09$ \\
NO5 & $6.23 \pm 0.73$ & $1.65 \pm 0.42$ & $1.98 \pm 0.52^{*}$ & $0.16 \pm 0.05$ & $0.86 \pm 0.17$ & $0.46 \pm 0.12$ & $0.31 \pm 0.11$ \\
PS5 & $5.98 \pm 0.43$ & $1.61 \pm 0.41$ & $2.66 \pm 0.72$ & $0.18 \pm 0.12$ & $0.97 \pm 0.25$ & $0.47 \pm 0.11$ & $0.30 \pm 0.12$ \\
SNO5 & $6.25 \pm 0.30$ & $1.58 \pm 0.57$ & $1.69 \pm 0.60^{*}$ & $0.15 \pm 0.05$ & $0.67 \pm 0.23$ & $0.54 \pm 0.10$ & $0.27 \pm 0.08$ \\
C10 & $7.14 \pm 0.59$ & $1.64 \pm 0.44$ & $3.66 \pm 1.30$ & $0.21 \pm 0.12$ & $0.76 \pm 0.25$ & $0.45 \pm 0.06$ & $0.25 \pm 0.06$ \\
NO10 & $7.16 \pm 0.46 \dagger$ & $1.63 \pm 0.50$ & $3.08 \pm 0.70 \dagger$ & $0.16 \pm 0.05$ & $0.80 \pm 0.14$ & $0.53 \pm 0.08$ & $0.32 \pm 0.06$ \\
PS10 & $6.65 \pm 0.88$ & $1.71 \pm 0.38$ & $2.72 \pm 0.99$ & $0.20 \pm 0.09$ & $0.98 \pm 0.19$ & $0.52 \pm 0.13$ & $0.34 \pm 0.13$ \\
SNO10 & $6.74 \pm 0.75$ & $1.71 \pm 0.46$ & $2.53 \pm 1.09$ & $0.25 \pm 0.09$ & $0.87 \pm 0.23$ & $0.52 \pm 0.09$ & $0.26 \pm 0.06$ \\
\hline
\end{tabular}

W/D, lung wet/dry weight; MDA, malondialdehyde; MPO, myeloperoxidase; iNOS, inducible NOS; cNOS, constitutive NOS; Vv, volume density of aerated alveoli; CV (Vv), the coefficient of variation of Vv. ${ }^{*} p<0.01$ vs. C5; $\uparrow p<0.05$ vs. NO5. 

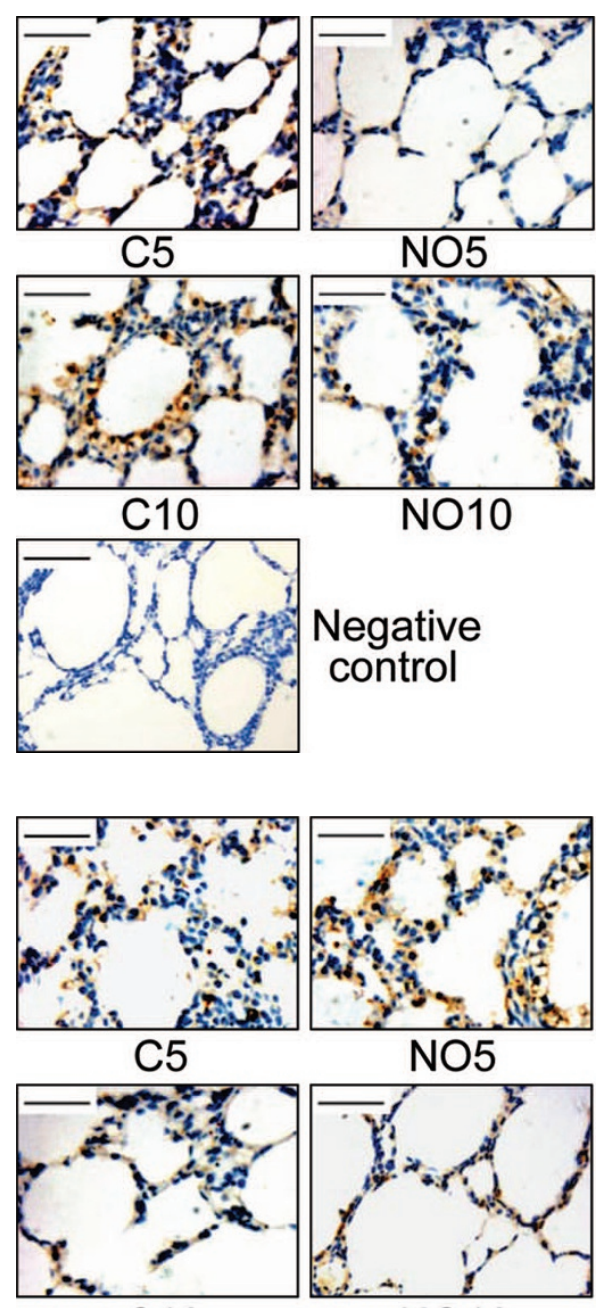

C10
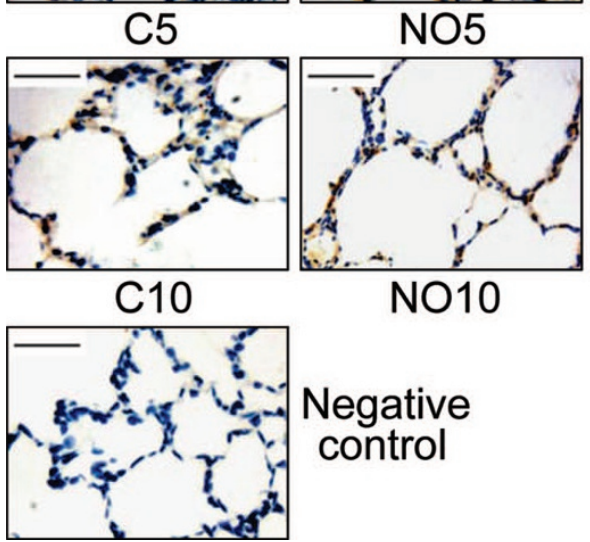

NO10

\section{Negative} control
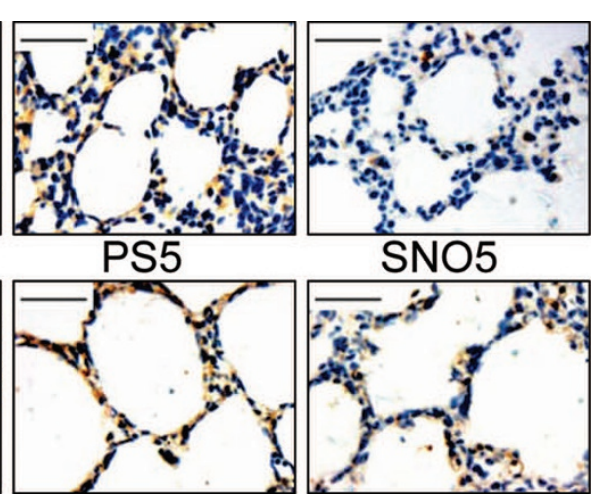

PS10

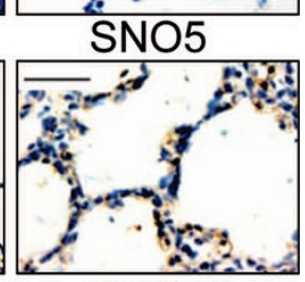

SNO10

Figure 4. Immunostaining for PDGF-B in the premature piglet lungs. Subgroup definitions see Figure 1 legends. Scale bar $=25 \mu \mathrm{m}$. Original magnifications, $400 \times$.

NO10 and SNO10 had lower IL-8 mRNA expression than C10 and PS10 (Fig. 6C). No significant differences of expression of TNF- $\alpha$ mRNA were found among the PEEP 5 and PEEP 10 subgroups (Fig. $6 D$ ), and of IL-8 and TNF- $\alpha$ mRNA between the PEEP 5 and PEEP 10 subgroups with the same treatment (Fig. 6C and $D$ ).

Again, there was decreased PDGF-B, in contrast to enhanced IGF-I, mRNA expression in NO5, NO10, SNO5, and SNO10 compared with C5, C10, PS5, and PS10 (Fig. $7 A$ and $B$ ). Significant difference was seen in NO5 and SNO5 compared with C5 or PS5 $(p<0.01)$. The PEEP 10 subgroups had enhanced expression of PDGF-B than in the corresponding PEEP 5 ones (Fig. 7A), especially between NO5 and NO10 $(p<0.01)$, SNO5 and SNO10 $(p<0.01)$ whereas the expression of IGF-I mRNA was opposite to that of the PDGF-B ( $p<0.05$, Fig. $7 B)$.

For the expression of KGF, HGF, VEGF, and TGF- $\beta 1$, no significant changes were found in both PEEP 5 and PEEP 10 subgroups (Fig. $7 C-F$ ).
Correlation analysis of the parameters. Correlation analyses of proinflammatory cytokine, GFs, oxygenation and lung mechanics are shown in Table 4. Expressions of IL-1 $\beta$, IL-6, mRNA were closely correlated to PDGF-B and MPO, but inversely to IGF-I, $\mathrm{PaO}_{2} / \mathrm{FiO}_{2}$ and Cdyn at $6 \mathrm{~h}$, and that of IL-8 to $\mathrm{KGF}$ and TGF- $\beta 1$. Expression of IGF-I mRNA was correlated to $\mathrm{PaO}_{2} / \mathrm{FiO}_{2}$, whereas PDGF-B was inversely correlated to $\mathrm{PaO}_{2} /$ $\mathrm{FiO}_{2}$, and that of IGF-I and KGF to Cdyn. Expression of PDGF-B mRNA was inversely correlated to IGF-I, and that of VEGF was correlated to TGF- $\beta 1$. A close correlation between $\mathrm{PaO}_{2} / \mathrm{FiO}_{2}$ and MPO was also found.

\section{DISCUSSION}

Inflammatory injury of premature lung is common for newborns subjected to hyperoxic condition and/or mechanical ventilation, and may be attenuated by protective ventilation strategy such as application of PEEP (1). In addition, pulmonary surfactant and iNO therapies are the important treatment 

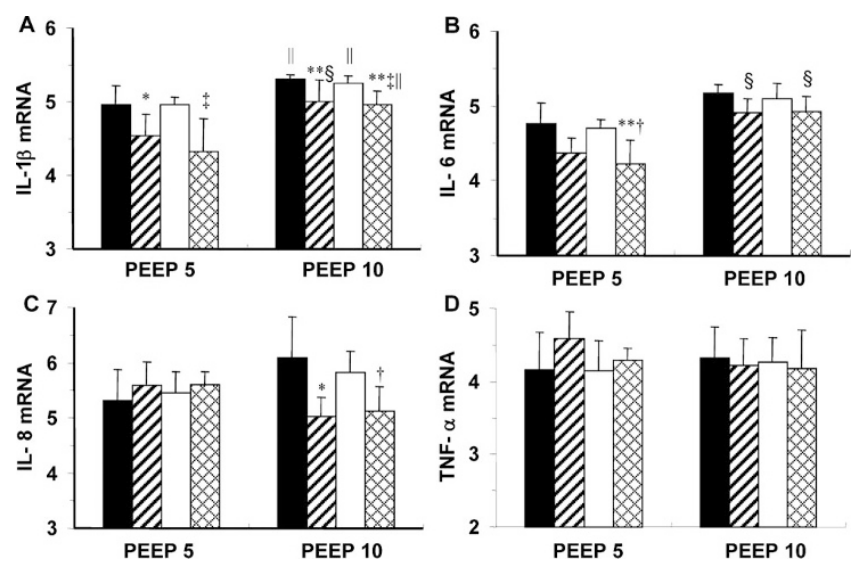

Figure 6. Expression of proinflammatory cytokine mRNA in lung tissues of PEEP 5 and PEEP 10 subgroups. Values are expressed in means and SD of common logarithm from mRNA copy numbers $(n=6)$. A, IL- $1 \beta ; B$, IL-6; $C$, IL-8; $D$, TNF- $\alpha$. Subgroup definitions see Fig. 1 legends. Subgroup symbols:

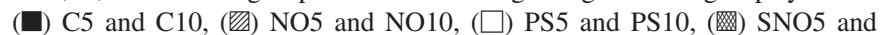
SNO10. In PEEP $5,{ }^{*} p<0.05,{ }^{* *} p<0.01$ vs. C5 and $\uparrow p<0.05, \$ p<0.01$ vs. PS5; in PEEP $10, * p<0.05, * * p<0.01$ vs. C10 and $\uparrow p<0.05, \ddagger p<0.01$ vs. PS10; $\S p<0.05, \| p<0.01$ vs. the corresponding ones in PEEP 5.

of hypoxic respiratory failure in preterm lungs. However, much less information is available about expression of proinflammatory cytokines and GFs in relation to ventilated modalities, surfactant, and iNO.

Studies showed that iNO decreased early lung inflammation (8-9), maintained surfactant activity (7) and improved lung structure in animal models of bronchopulmonary dysplasia $(10,16-17)$. PDGF-B and IGF-I are the important GFs in lung development (18). PDGF-B is a chemotactic factor for monocytes and granulocytes during inflammation and overexpression of PDGF-B induce inflammatory injury (19), whereas IGF signaling pathway plays a critical role in proliferation and differentiation of alveolar epithelium during tissue remodeling and repair (20). In this study results, iNO tended to be optimal in improvement of oxygenation, lower expression of IL-1 $\beta$, IL-6 and PDGF-B mRNA, and enhanced
Table 4. Correlation analysis of the major findings from physiological and biochemical analysis

\begin{tabular}{|c|c|c|c|c|c|}
\hline & IL- $1 \beta$ & IL-6 & IL-8 & $\mathrm{PaO}_{2} / \mathrm{FiO}_{2}$ & Cdyn \\
\hline \multicolumn{6}{|c|}{ PDGF-B } \\
\hline $\mathrm{r}$ & 0.571 & 0.585 & 0.230 & -0.576 & -0.303 \\
\hline$p$ & $3.38 \mathrm{e}-005$ & $1.93 \mathrm{e}-005$ & 0.153 & $1.94 \mathrm{e}-004$ & 0.069 \\
\hline \multicolumn{6}{|c|}{ IGF-I } \\
\hline $\mathrm{r}$ & -0.794 & -0.782 & -0.033 & 0.379 & 0.499 \\
\hline$p$ & $7.80 \mathrm{e}-011$ & $2.17 \mathrm{e}-010$ & 0.839 & 0.021 & 0.002 \\
\hline \multicolumn{6}{|c|}{ KGF } \\
\hline $\mathrm{r}$ & -0.271 & -0.247 & 0.629 & 0.145 & 0.542 \\
\hline$p$ & 0.068 & 0.097 & $1.40 \mathrm{e}-005$ & 0.393 & 0.001 \\
\hline \multicolumn{6}{|c|}{ TGF- $\beta 1$} \\
\hline $\mathrm{r}$ & -0.238 & -0.143 & 0.489 & -0.173 & 0.043 \\
\hline$p$ & 0.104 & 0.333 & 0.001 & 0.293 & 0.794 \\
\hline \multicolumn{6}{|c|}{$\mathrm{PaO}_{2} / \mathrm{FiO}_{2}$} \\
\hline $\mathrm{r}$ & -0.408 & -0.511 & -0.044 & 1.000 & 0.436 \\
\hline$p$ & 0.010 & 0.001 & 0.809 & - & 0.006 \\
\hline \multicolumn{6}{|c|}{ Cdyn } \\
\hline $\mathrm{r}$ & -0.376 & -0.496 & 0.235 & 0.436 & 1.000 \\
\hline$p$ & 0.018 & 0.001 & 0.188 & 0.006 & - \\
\hline \multicolumn{6}{|c|}{ MPO } \\
\hline $\mathrm{r}$ & 0.537 & 0.503 & -0.097 & -0.500 & -0.240 \\
\hline$p$ & $8.23 \mathrm{e}-005$ & $2.70 \mathrm{e}-004$ & 0.547 & 0.001 & 0.142 \\
\hline
\end{tabular}

KGF, keratinocyte growth factor; Cdyn, dynamic compliance; MPO, myeloperoxidase.

expression of IGF-I mRNA by 5- to 10-fold real difference in ventilated preterm piglet lungs at early postnatal life, which should have alleviated inflammatory injury and contributed to lung repair. The exact mechanism regulating GFs and cytokines, and their causal relationship needs to be further investigated. iNO transiently affects de novo synthesis of surfactant phospholipids in vivo with hyperoxic and endotoxic lung injury $(21,22)$, but this effect was not found in this study.

In the present study, it also showed that, compared with 10-12 $\mathrm{cm} \mathrm{H}_{2} \mathrm{O}$ PEEP, 5-6 cm $\mathrm{H}_{2} \mathrm{O}$ PEEP was beneficial as the effects of iNO on oxygenation improvement, decreased proinflammatory cytokines and enhanced PDGF-B in contrast to suppressed IGF-I. Berg et al. (23) provided additional
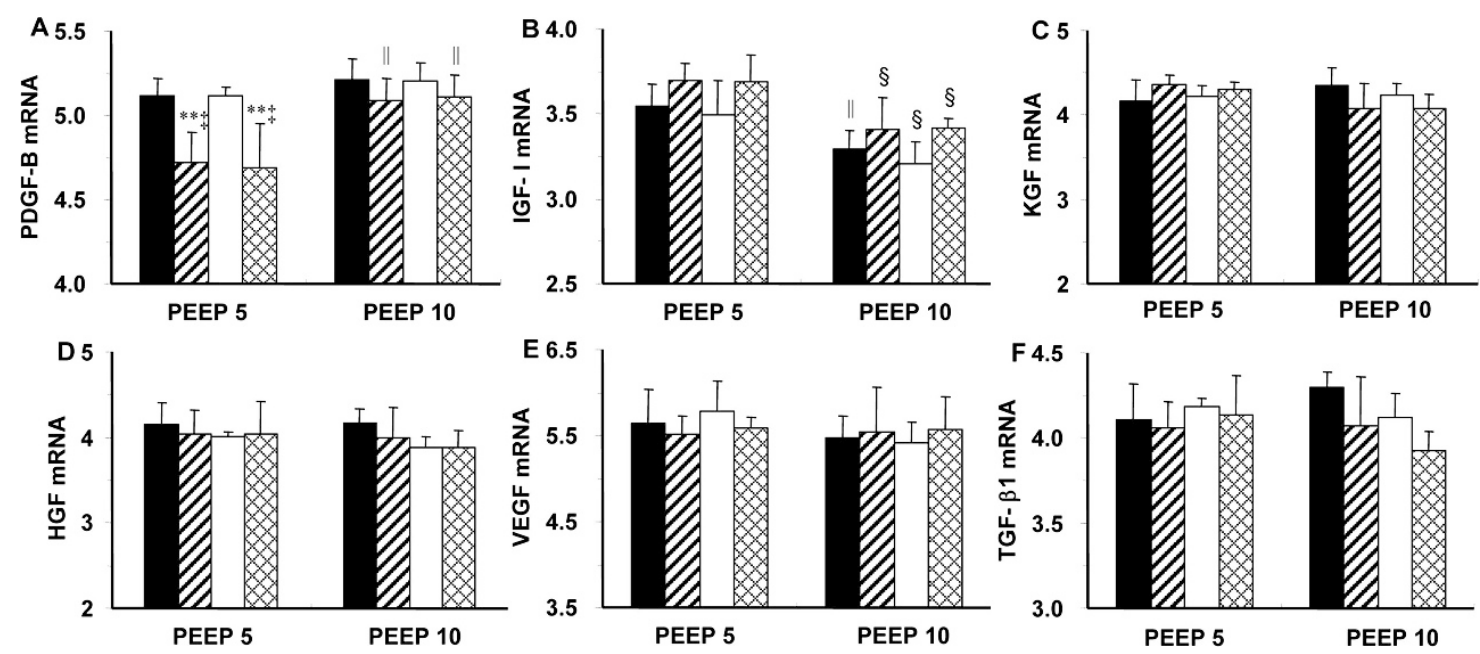

Figure 7. Expression of growth factor mRNA in lung tissues of PEEP 5 and PEEP 10 subgroups. Values are expressed in means and SD of common logarithm from mRNA copy numbers $(n=6)$. A, PDGF-B; $B$, IGF-I; $C$, keratinocyte growth factor (KGF); $D$, hepatocyte growth factor (HGF); $E$, VEGF; $F$, TGF- $\beta 1$. Subgroup definitions see Figure 1 legends. Subgroup symbols see Figure 6. In PEEP 5, ** $p<0.01 v s$. C5 and $\ddagger p<0.01 v s$. PS5; $\$ p<0.05$, $\| p<0.01 v s$. the corresponding ones in PEEP 5. 
evidence that high lung inflation induced by high PEEP $(9 \mathrm{~cm}$ $\mathrm{H}_{2} \mathrm{O}$ ) affects expression of GFs mRNA, which is related to remodeling of extracellular matrix in lung parenchyma. These results implied that application of PEEP might affect injury-repair response and remodeling because of differential expressions of these GFs and cytokines. Michna et al. (24) found that, in surfactant-treated, ventilated preterm lambs, increasing PEEP $(0$, $4,7 \mathrm{~cm} \mathrm{H}_{2} \mathrm{O}$ ) enhances alveolar pool size and large aggregate of surfactant phospholipids from BALF by 3- to 4-folds along with improved oxygenation and lung compliance. However, in this study, 10-12 PEEP $\mathrm{cm} \mathrm{H}_{2} \mathrm{O}$ did not increase endogenous surfactant pool size compared with 5-6 PEEP $\mathrm{cm} \mathrm{H}_{2} \mathrm{O}$.

In the present study, exogenous surfactant treatment did not improve oxygenation and affect the expression of proinflammatory cytokines and GFs. We speculated the preterm piglets might have intrapulmonary shunting because of pulmonary arterial hypertension and extrapulmonary shunting from PDA opening, thus the piglets lacked the response of oxygenation improvement to surfactant alone. A study performed on isolated and perfused rat lungs seemed to indicate that surfactant was able to induce increased synthesis and release of proinflammatory cytokines (25). In contrast, human studies suggested that surfactant therapy reduced the proinflammatory mediators (26). This differential response to surfactant therapy might be related to the species difference, lung maturation status, and the way of experiment.

The major features of expression of GF mRNA suggested that PDGF-B and IGF-I, though expressed in opposite ways, were two sensitive responders to the ventilator settings and added iNO in early postnatal life of the immature piglet lungs. Moreover, our results showed that there was correlation between mRNA expressions of proinflammatory cytokines and GFs. These findings suggested GFs might play pivotal roles in interaction with ventilator-induced cascade of major inflammatory cytokine expression. It was unclear whether these relationships were beneficial to alveolar epithelial cell repair and regeneration, or detrimental in subsequent alveolarization. However, our results supported that the adequate use of respiratory therapies should affect the expression in both GFs and cytokines in early postnatal life of the immature lung. Put together, interactions among PEEP, surfactant, and iNO in our settings explained potential as well as limitation of combined respiratory therapies in lung protective ventilation. As a limitation, these results might not be generalized to extremely low birth weight infants in humans.

In conclusion, we used a preterm piglet model and tested protective ventilation strategy on expressions of proinflammatory cytokines and GFs. Both PDGF-B and IGF-I had different expression patterns in the early stage of ventilator-associated lung inflammatory injury, and adequate PEEP with iNO may mitigate the lung inflammation, and should be given according to lung maturation. Further studies of temporal expression patterns of these cytokines and GFs are needed to elucidate the mechanisms and causal relationship from early to later postnatal life.
Acknowledgments. The authors thank Drs. Dinghai Fang for the animal experiment and Mrs Fengfei Xu for technical assistance in lung sample process.

\section{REFERENCES}

1. Clark RH, Slutsky AS, Gerstmann DR 2000 Lung protective strategies of ventilation in the neonate: what are they? Pediatrics 105:112-114

2. Bourbon J, Boucherat O, Chailley-Heu B, Delacourt C 2005 Control mechanisms of lung alveolar development and their disorders in bronchopulmonary dysplasia. Pediatr Res 57:38R-46R

3. Ozdemir A, Brown MA, Morgan WJ 1997 Markers and mediators of inflammation in neonatal lung disease. Pediatr Pulmonol 23:292-306

4. Jobe AH, Bancalari E 2001 Bronchopulmonary dysplasia. Am J Respir Crit Care Med 163:1723-1729

5. Warburton D, Schwarz M, Tefft D, Flores-Delgado G, Anderson KD, Cardoso WV 2000 The molecular basis of lung morphogenesis. Mech Dev 92:55-81

6. Naik AS, Kallapur SG, Bachurski CJ, Jobe AH, Michna J, Kramer BW, Ikegami M 2001 Effects of ventilation with different positive end-expiratory pressure on cytokine expression in the preterm lamb lung. Am J Respir Crit Care Med 164:494-498

7. Ballard PL, Gonzales LW, Godinez RI, Godinez MH, Savani RC, McCurnin DC, Gibson LL, Yoder BA, Kerecman JD, Grubb PH, Shaul PW 2006 Surfactant composition and function in a primate model of infant chronic lung disease: effects of inhaled nitric oxide. Pediatr Res 59:157-162

8. Kinsella JP, Parker TA, Galan H, Sheridan BC, Halbower AC, Abman SH 1997 Effects of inhaled nitric oxide on pulmonary edema and lung neutrophil accumulation in severe experimental hyaline membrane disease. Pediatr Res 41:457-463

9. Raychaudhuri B, Dweik R, Connors MJ, Buhrow L, Malur A, Drazba J, Arroliga AC, Erzurum SC, Kavuru MS, Thomassen MJ 1999 Nitric oxide blocks nuclear factorkappaB activation in alveolar macrophages. Am J Respir Cell Mol Biol 21:311-316

10. McCurnin DC, Pierce RA, Chang LY, Gibson LL, Osborne-Lawrence S, Yoder BA, Kerecman JD, Albertine KH, Winter VT, Coalson JJ, Crapo JD, Grubb PH, Shaul PW 2005 Inhaled NO improves early pulmonary function and modifies lung growth and elastin deposition in a baboon model of neonatal chronic lung disease. Am J Physiol Lung Cell Mol Physiol 288:L450-L459

11. ter Horst SA, Walther FJ, Poorthuis BJ, Hiemstra PS, Wagenaar GT 2007 Inhaled nitric oxide attenuates pulmonary inflammation and fibrin deposition and prolongs survival in neonatal hyperoxic lung injury. Am J Physiol Lung Cell Mol Physiol 293:L35-L44

12. Zhu Y, Guo C, Cao L, Gong X, Wang C, Sun B 2005 Different effects of surfactant and inhaled nitric oxide in modulation of inflammatory injury in ventilated piglet lungs. Pulm Pharmacol Ther 18:303-313

13. Zhou ZH, Sun B, Lin K, Zhu L 2000 Prevention of rabbit acute lung injury by surfactant, inhaled nitric oxide and pressure support ventilation. Am J Respir Crit Care Med 161:581-588

14. Wang X, Sun Z, Qian L, Guo C, Yu W, Wang W, Lu KW, Taeusch HW, Sun B 2006 Effects of hyaluronan-fortified surfactant in ventilated premature piglets with respiratory distress. Biol Neonate 89:15-24

15. Mehta S, Lilly CM, Rollenhagen JE, Haley KJ, Asano K, Drazen JM 1997 Acute and chronic effects of allergic airway inflammation on pulmonary nitric oxide production. Am J Physiol Lung Cell Mol Physiol 272:L124-L131

16. Bland RD, Albertine KH, Carlton DP, MacRitchie AJ 2005 Inhaled nitric oxide effects on lung structure and function in chronically ventilated preterm lambs. Am J Respir Crit Care Med 172:899-906

17. Lin YJ, Markham NE, Balasubramaniam V, Tang JR, Maxey A, Kinsella JP, Abman SH 2005 Inhaled nitric oxide enhances distal lung growth after exposure to hyperoxia in neonatal rats. Pediatr Res 58:22-29

18. Kumar VH, Ryan RM 2004 Growth factors in the fetal and neonatal lung. Front Biosci 9:464-480

19. Siegbahn A, Hammacher A, Westermark B, Heldin CH 1990 Differential effects of the various isoforms of platelet-derived growth factor on chemotaxis of fibroblasts, monocytes and granulocytes. J Clin Invest 85:916-920

20. Narasaraju TA, Chen H, Weng T, Bhaskaran M, Jin N, Chen J, Chen Z, Chinoy MR, Liu L 2006 Expression profile of IGF system during lung injury and recovery in rats exposed to hyperoxia: a possible role of IGF-1 in alveolar epithelial cell proliferation and differentiation. J Cell Biochem 97:984-998

21. Hu X, Guo C, Sun B 2007 Inhaled nitric oxide attenuates hyperoxic and inflammatory injury without alteration of phosphatidylcholine synthesis in rat lungs. Pulm Pharmacol Ther 20:75-84

22. Gong X, Guo C, Huang S, Sun B 2006 Inhaled nitric oxide alleviates hyperoxia suppressed phosphatidylcholine synthesis in endotoxin-induced injury in mature rat lungs. Respir Res 7:5

23. Berg JT, Fu Z, Breen EC, Tran HC, Mathieu-Costello O, West JB 1997 High lung inflation increases mRNA levels of ECM components and growth factors in lung parenchyma. J Appl Physiol 83:120-128

24. Michna J, Jobe AH, Ikegami M 1999 Positive end-expiratory pressure preserves surfactant function in preterm lambs. Am J Respir Crit Care Med 160:634-639

25. Welk B, Malloy JL, Joseph M, Yao LJ, Veldhuizen AW 2001 Surfactant treatment for ventilation-induced lung injury in rats: effects on lung compliance and cytokines. Exp Lung Res 27:505-520

26. Tegtmeyer FK, Moller J, Zabel P 2002 Inhibition of granulocyte activation by surfactant in a 2-yr-old female with meningococcus-induced ARDS. Eur Respir J 19:776-779 